\title{
VIGILÂNCIA SANITÁRIA ESTADUAL E A RESPONSABILIDADE PELO ESTOQUE DE MEDICAMENTOS CONTROLADOS DE USO HUMANO UTILIZADOS EM ESTABELECIMENTO VETERINÁRIO: análise da legislação
}

\section{State Sanitary Surveillance and responsibility for the stock of controlled medicines for human use used in veterinarian establishments: legislation analysis}

\author{
Angela Simonetti ${ }^{1 *}{ }^{(\mathbb{D}}$. \\ *Autor Correspondente: Angela Simonetti, Rua Ribeiro do Vale, 800, Brooklin, \\ São Paulo, SP, Brasil. CEP: 04568-002. \\ E-mail: gelasimonetti@hotmail.com
}

Como citar: SIMONETTI, A. Vigilância Sanitária Estadual e a responsabilidade pelo estoque de medicamentos
controlados de uso humano utilizados em estabelecimento veterinário: análise da legislação. Revista de Educação Continuada em Medicina Veterinária e Zootecnia do CRMV-SP, São Paulo, v. 19, n. 1, 2021. DOI: https://doi.org/10.36440/recmvz.v19i1.38222.

Cite as: SIMONETTI, A. State Sanitary Surveillance and responsibility for the stock of controlled medicines for human use used in veterinarian establishments: legislation analysis. Journal of Continuing Education in Veterinary Medicine and Animal Science of CRMV-SP, São Paulo, v. 19 , n. 1, 2021. DOI: https://doi. org/10.36440/recmvz.v19i1.38222.

\section{Resumo}

Para fins de licenciamento sanitário, a portaria do Centro de Vigilância Sanitária Estadual de São Paulo, CVS no 01/2020, classifica a Atividade Veterinária (CNAE 7500-1/00) como de alto risco no caso em que o estabelecimento mantenha estoque de medicamentos de uso humano para utilização em animais atendidos no local. Nessa situação, exige-se a presença de profissional responsável técnico pelo funcionamento do estoque de medicamentos, denominando-o de dispensário. Quando nesse estoque estiver incluída a guarda e fornecimento de medicamentos controlados (entorpecentes e psicotrópicos) de uso humano, a Vigilância Sanitária Estadual interpreta e orienta as Vigilâncias Municipais, que de acordo com a Portaria SVS MS no 344/1998 apenas o profissional farmacêutico está habilitado a responder

1 Médica-veterinária pela Universidade São Paulo (USP), advogada, mestre em Direito Político e Econômico pela Universidade Mackenzie, com especialização em Saúde Pública e Direito Sanitário, pela USP, Faculdade de Saúde Pública, São Paulo, SP, Brasil 
pelo dispensário, não reconhecendo a competência do profissional médico-veterinário como responsável pela guarda desses medicamentos no estabelecimento veterinário. Esse tema não está nacionalmente regulamentado, daí a importância de demonstrar a competência legal do médico-veterinário sobre a guarda de medicamentos controlados de uso humano, necessários para fins terapêuticos, clínicos ou diagnósticos realizados no estabelecimento veterinário.

Palavras-chave: Responsabilidade Técnica Veterinária. Guarda de Medicamentos Controlados.

\section{Abstract}

For health licensing in the state of São Paulo, Brazil, the Veterinary Activity (CNAE 7500-1/00) is classified as being of high risk when the establishment keeps stock of human medicines used in animals treated in the place. In this situation, the presence of a technician responsible for what they consider to be a dispensary is required and, the State Health Surveillance consider that only a pharmaceutical professional is qualified for the custody and supply of human controlled medicines, and do not recognize the competence of the veterinary professional as responsible for the safekeeping these drugs in the veterinary establishment. This issue is not legally pacified; hence the importance of demonstrating the legal competence of the veterinarian on the safekeeping of medicines for human use, that the presence of a pharmacist cannot be required for veterinary establishment, even when there is a stock of human controlled medicines for therapeutic, clinical, or diagnostic purposes performed there.

Keywords: Veterinarian Responsibility. Guarding of Controlled Medications.

\section{Introdução}

A atual legislação sanitária estadual de São Paulo, Portaria do Centro de Vigilância Sanitária, CVS no 1/2019 (SÃO PAULO, 2019), dispõe em seu artigo 17 que o estabelecimento veterinário não é sujeito à Vigilância Sanitária, porém, caso possua estoque de medicamento de uso humano, será sujeito a licenciamento sanitário por exercer atividade de dispensário de medicamentos de uso humano. Assim, a Atividade Veterinária (CNAE 7500-1/00) passa a ser classificada como sendo de alto risco, sob a égide da Vigilância Sanitária, quando o estabelecimento mantém estoque de medicamentos fabricados com a finalidade de uso humano para utilização em animais atendidos no local:

Art. 17 Compete à vigilância sanitária emitir, no âmbito das atividades veterinárias (CNAE 7500-1/00), Licença Sanitária (LS) apenas para as fontes de radiação ionizante (Anexo II) e para o dispensário de medicamentos de uso humano. (SÃO PAULO, 2019, p. 22)

Nessa situação, exige-se a presença de profissional responsável técnico pelo que consideram ser um dispensário e, apesar de não definir que deva ser farmacêutico quando se tratar de medicamento sujeito à Portaria no 344/1998 (BRASIL, 1998), há o entendimento de que apenas o profissional farmacêutico está habilitado a responder pela guarda e fornecimento de medicamentos controlados de uso humano e não se reconhece a competência do profissional médico-veterinário como responsável pela guarda desses medicamentos no estabelecimento veterinário.

\section{Justificativa}

Consideramos existir um equívoco de interpretação sobre as exigências legais para tal atividade, especialmente com relação ao profissional habilitado a responder tecnicamente por ela. Este tema não está pacificado no território nacional já que não há uma legislação específica. A Agência 
Nacional de Vigilância Sanitária (Anvisa), chegou a publicar uma referência técnica sobre o assunto (AGÊNCIA NACIONAL DE VIGILÂNCIA SANITÁRIA, 2010), no qual menciona que o armazenamento dos medicamentos controlados deve ser em armário ou sala de acesso restrito, com chave, sob guarda do médico-veterinário responsável, mas o documento não tem qualquer poder legal, sendo apenas material de referência para que estados e municípios elaborem e instituam legislações locais a respeito do assunto tratado. 0 município de São Paulo tem a sua própria legislação (SÃO PAULO, 2016), mas a maioria dos demais municípios paulistas seguem a portaria estadual e as orientações do CVS. Daí a importância de abordarmos essa questão, defendendo a tese de que não se pode exigir a presença de farmacêutico para o funcionamento do estabelecimento veterinário, mesmo quando haja estoque de medicamentos controlados de uso humano para fins terapêuticos, clínicos ou diagnósticos em animais.

Nosso objetivo é analisar a legislação, a fim de reconhecer a legitimidade do médico-veterinário como profissional responsável técnico pelos medicamentos de uso humano, utilizados no estabelecimento veterinário, incluindo os de controle especial.

\section{Metodologia}

Para tanto utilizaremos a metodologia da análise gramatical, lógica e sistemática das normas relacionadas à Responsabilidade Técnica e sobre guarda de medicamentos de uso humano utilizados por estabelecimento veterinário.

Inicialmente, vejamos o ensinamento do Professor Adriano Ferreira (FERREIRA, 2018) em seu artigo Métodos e Tipos de Interpretação, no qual explica que a boa interpretação da norma legal deve esclarecer seu significado, mostrando sua validade, seu alcance social, demonstrando que o conflito pode ser resolvido conforme os fins sociais da norma e a concretização de valores que levam ao bem comum. Para resolver o problema do significado e da validade da norma, existem os métodos de interpretação gramatical, lógica e sistemática. A gramatical desvenda o significado da norma, enfrentando dificuldades léxicas e de relações entre as palavras. A interpretação lógica resolve contradições entre termos numa norma jurídica, chegando-se a um significado coerente. A sistemática, por sua vez, confronta normas jurídicas entre si. Pressupondo que o ordenamento é um todo unitário, sem incompatibilidades, permite escolher o significado da norma que seja coerente com o conjunto. 0 método sistemático impede que as normas jurídicas sejam interpretadas de modo isolado, exigindo que o conjunto seja analisado simultaneamente à interpretação de qualquer texto normativo. Para demonstrar o alcance da norma legal, devemos precisar os fatos a que ela se refere. Após a determinação de um significado válido para a norma e de se encontrar os fatos a que se refere, resta mostrar que sua aplicação concretizará seus fins sociais e levará ao bem comum.

\section{Discussão}

No caso em questão, nota-se que a atividade licenciada é o estabelecimento veterinário que, para sua atividade de atendimento aos animais, necessita manter estoque de medicamentos. Por questões de mercado, e não sanitárias ou técnicas, alguns medicamentos necessários ao tratamento veterinário não são fabricados por laboratórios da área de saúde animal, mas por laboratórios farmacêuticos da área de saúde humana.

Verifica-se, na prática, que não existem disponíveis no mercado as formulações veterinárias de todos os medicamentos utilizados na Medicina Veterinária e, assim, o profissional se vê forçado a adquirir medicamento produzido para uso humano.

o fato de o medicamento ter sido fabricado para uso humano não altera em nada a sua 
farmacologia. Quando o mesmo, apesar de ter sido fabricado para uso humano, for destinado a animal, se torna produto de uso veterinário, conforme dispõe o Decreto Federal no 5.053/04, em seu artigo 20 - A, XX (BRASIL, 2004, p. 1):

XX - produto veterinário - toda substância química, biológica, biotecnológica ou preparação manufaturada cuja administração seja aplicada de forma individual ou coletiva, direta, misturada com os alimentos, destinada a prevenção, ao diagnóstico, à cura ou ao tratamento das doenças dos animais, incluindo os aditivos, suplementos promotores, melhoradores da produção animal, medicamentos, vacinas, antissépticos, desinfetantes de ambientes e de equipamentos, pesticidas e todos os produtos que, utilizados nos animais ou no seu habitat, protejam, restaurem ou modifiquem suas funções orgânicas e fisiológicas, ou também os produtos destinados ao embelezamento dos animais (redação dada pelo Decreto no 8.840 de 2016). (BRASIL, 2004, p. 2)

Assim sendo, mesmo que o medicamento tenha sido desenvolvido e produzido para uso humano, ao ser aplicado a animais passa a ser considerado produto veterinário e daí sua aplicação deverá ser realizada exclusivamente por médico-veterinário, conforme dispõe a Lei no 5.517/ 1968, artigo 50, alínea "a" (BRASIL, 1968, p. 9401):

Art. 50 - É da competência privativa do médico-veterinário o exercício das seguintes atividades e funções a cargo da União, dos Estados, dos Municípios, dos Territórios Federais, entidades autárquicas, paraestatais de economia mista e particulares: Prática clínica em todas as suas modalidades. (BRASIL, 1968, p. 9401)

Nesse sentido, é privativa do médico-veterinário a responsabilidade técnica pela guarda dos medicamentos utilizados na prática clínica no estabelecimento veterinário.

A própria definição de medicamento na Portaria no 344/1998 não diferencia o humano do veterinário: Medicamento - Produto farmacêutico, tecnicamente obtido ou elaborado, com finalidade profilática, curativa, paliativa ou para fins de diagnóstico.

Outra questão importante a ser analisada é a definição de dispensário de medicamentos. A Lei no 5991/1973, em seu Art. 30, estabelece: "XIV - Dispensário de medicamentos - setor de fornecimento de medicamentos industrializados, privativo de pequena unidade hospitalar ou equivalente." (BRASIL, 1973, p. 13049).

O que, claramente, não é o caso do estabelecimento veterinário. Nele, não há um setor de fornecimento de medicamentos, mas a guarda dos mesmos pelo profissional que os administra durante sua consulta em procedimento clínico, diagnóstico ou cirúrgico.

Isso é válido também em relação aos medicamentos psicotrópicos e aos demais sujeitos à Portaria SMS/MS no 344, de 12 de maio de 1998, que dispõe sobre a aprovação do Regulamento Técnico sobre substâncias e medicamentos sujeitos a controle especial. Nela se determina a necessidade de licença sanitária para a seguinte situação:

Licença de Funcionamento - Permissão concedida pelo órgão de saúde competente dos Estados, Municípios e Distrito Federal, para o funcionamento de estabelecimento vinculado a empresa que desenvolva qualquer das atividades enunciadas no artigo $2 \circ$ deste Regulamento Técnico.

Art. 20 Para extrair, produzir, fabricar, beneficiar, distribuir, transportar, preparar, manipular, fracionar, importar, exportar, transformar, embalar, reembalar, para qualquer 
fim, as substâncias constantes das listas deste Regulamento Técnico (ANEXO I) e de suas atualizações, ou os medicamentos que as contenham, é obrigatória a obtenção de Autorização Especial concedida pela Secretaria de Vigilância Sanitária do Ministério da Saúde. (BRASIL, 1998, p. 3)

Ou seja, não é prevista uma licença sanitária quando da guarda e uso de substâncias e medicamentos sujeitos a controle especial. Ressaltando que este uso é inerente à atividade médica-veterinária.

Por outro lado, fica claro, na mesma Portaria, que deve haver escrituração em livro próprio quando do uso dessas substâncias ou medicamentos:

Art. 62. Todo estabelecimento, entidade ou órgão oficial que produzir, comercializar, distribuir, beneficiar, preparar, fracionar, dispensar, utilizar, extrair, fabricar, transformar, embalar, reembalar, vender, comprar, armazenar ou manipular substância ou medicamento de que trata este Regulamento Técnico e de suas atualizações, com qualquer finalidade deverá escriturar e manter no estabelecimento para efeito de fiscalização e controle, livros de escrituração conforme a seguir discriminado. (BRASIL, 1998, p. 25)

A Portaria SMS/MS no 344/1998 não deixa dúvidas de que sua regulamentação não se aplica a medicamentos de uso veterinário, regulamentados em legislação específica: "Art. 93. Os medicamentos destinados a uso veterinário serão regulamentados em legislação específica." (BRASIL, 1998, p. 33)

Portanto, utilizar da Portaria em questão para embasar a exigência de farmacêutico em estabelecimento veterinário, onde há estoque de medicamentos controlados destinados a uso veterinário, não é cabível. Quando a Portaria menciona, em seu artigo 67, a necessidade de farmacêutico responsável pela guarda dos medicamentos existentes nos estabelecimentos, entende-se que se aplica aos estabelecimentos de saúde humana e não os de saúde animal:

Art. 67. As substâncias constantes das listas deste Regulamento Técnico e de suas atualizações, bem como os medicamentos que as contenham, existentes nos estabelecimentos, deverão ser obrigatoriamente guardados sob chave ou outro dispositivo que ofereça segurança, em local exclusivo para este fim, sob a responsabilidade do farmacêutico ou químico responsável, quando se tratar de indústria farmoquímica. (BRASIL, 1998, p. 26)

Cabe ressaltar que o órgão competente para definir o exercício profissional e emitir a anotação de responsabilidade técnica é o Conselho de Classe. A Vigilância Sanitária exige a presença de Responsável Técnico para o licenciamento de determinadas atividades, mas não cabe a ela definir quem são os profissionais habilitados a essa função. Além disso, essa competência está definida em Lei e uma Portaria não tem o condão de alterar essa regra. Assim estabelece o artigo 10 da Lei no 6.839/1980 (BRASIL, 1980, p. 21881):

Art. 10 - 0 registro de empresa e anotação dos profissionais legalmente habilitados, delas encarregados, serão obrigatórios das entidades competentes para a fiscalização dos exercícios das diversas profissões, em razão da atividade básica ou em relação àquela pela qual prestam serviços a terceiros. (BRASIL, 1980, p. 21881) 


\section{Conclusão}

Da análise gramatical, lógica e sistemática da legislação referenciada, verifica-se que não há embasamento legal para, primeiro, considerar a guarda de medicamentos em estabelecimento veterinário como sendo atividade de dispensário e, segundo, exigir que o profissional responsável técnico pela guarda desses medicamentos, quando forem controlados, produzidos para uso humano, seja o farmacêutico.

A Vigilância Sanitária tem como finalidade a prevenção a riscos e a proteção da saúde humana. Deve atuar interpretando as normas de modo que o conjunto seja analisado, para que sua aplicação concretize os fins sociais e de saúde pública, e leve ao bem comum.

o fato de o estabelecimento veterinário necessitar adquirir, por questões de mercado, medicamentos, inclusive os de controle especial, produzidos para uso humano, não transforma sua atividade em dispensário de medicamentos. Por outro lado, quando o medicamento fabricado para uso humano é utilizado para tratamento animal, passa a ser medicamento de uso veterinário.

Além disso, conforme demonstrado, não há amparo legal para a exigência de que o estabelecimento veterinário contrate profissional farmacêutico como responsável técnico pelos medicamentos de controle especial de uso humano com base na Portaria no 344/1998, pois esta não trata de medicamentos destinados a uso veterinário que são regulamentados em legislação específica.

Ademais, a responsabilidade técnica é definida pelos Conselhos de classe, conforme previsão em Lei Federal, que não pode ser alterada por portaria.

\section{Referências}

AGÊNCIA NACIONAL DE VIGILÂNCIA SANITÁRIA. Referência técnica para o funcionamento dos serviços veterinários. 2010. Disponível em: https://www.crmv-pr.org.br/uploads/pagina/arquivos/ Referencia tecnica funcionamento servicos veterinarios.pdf. Acesso em: 7 out. 2021.

BRASIL. Congresso Nacional. Lei no 5.517, de 23 de outubro de 1968. Dispõe sobre o exercício da profissão de médico-veterinário e cria os Conselhos Federal e Regionais de Medicina Veterinária. Diário Oficial da União da República Federativa do Brasil, Brasília, 25 out. 1968.

BRASIL. Congresso Nacional. Lei no 5.991, de 17 de dezembro de 1973. Dispõe sobre o controle sanitário do comércio de drogas, medicamentos, insumos farmacêuticos e correlatos, e dá outras providências. Diário Oficial da União da República Federativa do Brasil, Brasília, 21 dez. 1973.

BRASIL. Congresso Nacional. Lei no 6.839, de 30 de outubro de 1980. Dispõe sobre o registro de empresas nas entidades fiscalizadoras do exercício de profissões. Diário Oficial da União da República Federativa do Brasil, Brasília, 3 nov. 1980.

BRASIL. Ministério da Saúde. Portaria SVS/MS no 344, de 12 de maio de 1998. Aprova o regulamento técnico sobre substâncias e medicamentos sujeitos a controle especial. Diário Oficial da União da República Federativa do Brasil, Brasília, p. 37, 19 maio 1998. Republicada no Diário Oficial da União da República Federativa do Brasil, Brasília, 10 fev. 1999.

BRASIL. Presidência da República. Decreto Federal no 5.053, de 22 de abril de 2004. Aprova 0 regulamento de fiscalização de produtos de uso veterinário e dos estabelecimentos que os fabriquem ou comerciem, e dá outras providências. Diário Oficial da União da República Federativa do Brasil, Brasília, 23 abr. 2004.

FERREIRA, A. Métodos e tipos de interpretação. 2018. Disponível em: https://direito.legal/ interpretacao-e-alicacao/51-metodos-e-tipos-de-interpretacao/. Acesso em: 10 set. 2020. 
SÃO PAULO. Guia técnico: portaria 641/2016-SMS.G: regulamento técnico sobre as condições higiênico-sanitárias e as boas práticas para estabelecimentos e serviços médico-veterinários. 2016. Disponível em: https://www.prefeitura.sp.gov.br/cidade/secretarias/upload/chamadas/guia_tecnico_ portaria veterinarias 1470426533.pdf. Acesso em: 7 out. 2021.

SÃo PAULO. Secretaria de Estado da Saúde. Coordenadoria de Controle de Doenças. Centro de Vigilância Sanitária. Portaria CVS no 1, de 09 de janeiro de 2019. Disciplina, no âmbito do Sistema Estadual de Vigilância Sanitária - Sevisa, o licenciamento dos estabelecimentos de interesse da saúde e das fontes de radiação ionizante, e dá providências correlatas. Diário Oficial do Estado de São

Paulo, São Paulo, seção 1, no 21, p. 50, 31 jan. 2019. 